\title{
The formation of zinc coatings in nanocrystallised zinc powders
}

\author{
Rashit Galin \\ vika-gal.chel@rambler.ru | (D) https://orcid.org/0000-0002-2679-0675 \\ Vika-Gal Co., Ltd, Chelyabinsk, Russia
}

Boris Khina

khina@tut.by | (D) https://orcid.org/0000-0002-5179-0941

Physico-technical Institute, National Academy of Sciences of Belarus, Minsk, Belarus

\author{
Natalia Shaburova \\ nata8855@gmail.com | (iD https://orcid.org/0000-0001-8339-8293 \\ South Ural State University (NRU), Chelyabinsk, Russia
}

\section{Anna Wassilkowska \\ awassilkowska@pk.edu.pl | (D) https://orcid.org/0000-0003-0453-3480 \\ Faculty of Environmental and Power Engineering, Cracow University of Technology, Poland}

\author{
Scientific Editor: Michat Zielina, Cracow \\ University of Technology \\ Technical Editor: Aleksandra Urzędowska, \\ Cracow University of Technology Press \\ Language Verification: Timothy Churcher, \\ Merlin Language Services \\ Typesetting: Anna Basista, Cracow \\ University of Technology Press
}

Received: March 8, 2021

Accepted: June 30, 2021

\begin{abstract}
Copyright: @ 2021 Galin, Khina, Shaburova, Wassilkowska. This is an open access article distributed under the terms of the Creative Commons Attribution License, which permits unrestricted use, distribution, and reproduction in any medium, provided the original author and source are credited.
\end{abstract}

Data Availability Statement: All relevant data are within the paper and its Supporting Information files.

Competing interests: The authors have declared that no competing interests exist.

Citation: Galin, R., Khina, B., Shaburova, N., Wassilkowska, A. (2021). The formation of zinc coatings in nanocrystallised zinc powders. Technical Transactions: e2021009. https://doi.org/10.37705/ TechTrans/e2021009

\begin{abstract}
The kinetics of and mechanism for galvanising low-carbon steel $(0.2 \% \mathrm{C})$ were examined in powder media which were pre-treated to obtain a fine nanostructured $\mathrm{ZnO}$ layer on the surface of zinc powder particles. The effective diffusion coefficient of $\mathrm{Zn}$ atoms through the $\mathrm{ZnO}$ shell was estimated to be in the order of $1 \cdot 10^{-10} \mathrm{~m}^{2} \cdot \mathrm{s}^{-1}$. The contribution of the $\mathrm{Zn}$-gas evaporation/condensation microprocesses, which could occur in relation to the above diffusion through a nanostructured surface layer, was evaluated with numerical calculations in the temperature range of 550-950 $\mathrm{K}$ and for an average particle size of $\mathrm{ZnO}$ up to $100 \mathrm{~nm}$. Our results suggest that the outward diffusion of metallic zinc takes place from the core of powder microparticles across the nano-grain boundaries of their modified surface layer, and can be further intensified by the presence of other inter-phase defects, such as nano-porosity.
\end{abstract}

Keywords: nanocrystallised powders; galvanising; zinc coatings; coating structure 


\section{Introduction}

In modern industry, an urgent problem is the corrosion control of machine parts and units subjected to corrosion attacks in hostile environments (Revie, Uhlig, 2008). One of the most widely used methods for corrosion protection in the automotive, oil, chemical and mining industries, offshore construction and other areas is the hot-dip galvanisation of steel bars, tubes, sheets and other products (Kuklik, Kudlacek, 2016). Notwithstanding the obvious advantages of this process, especially in mass production, it should be noted that it features several drawbacks including the high cost of spelter baths, the rather high service and maintenance expenses, the need for thorough surface preparation before processing, the dissolution of iron in molten zinc and the formation of dross which should be removed from the bath, the need for the withdrawal of toxic zinc vapours, and emerging ecological issues. At present, more interest is directed towards diffusion or galvanising processes in powder media, which have a long history of application in mass and especially small-batch manufacturing.

Currently, the most widespread method of diffusion galvanising in industry is the contact method, which involves the use of saturating mixtures consisting of zinc powder and inert materials, for example, aluminium oxide (Proskurkin, Gorbunov, 1975). This galvanising technology includes the obligatory introduction of a fresh portion of zinc powder into the saturating mixture before treatment. When saturating mixtures are repeatedly used with inert additives, there is a change in the chemical and granulometric size composition of both zinc and the inert component of the saturating mixtures, which negatively affects the consistency of the galvanising results. The difference in the density of the particles of zinc and inert components leads to delamination of the mixture during galvanising. This limits the dimensions of galvanising chambers and complicates the mechanisation of the process of separating galvanised elements and saturating mixtures upon the completion of galvanising. At the same time, the homogeneity of the coating and the ability to reproduce the results of galvanising are determined by the purity of the zinc powder, its granulometric size composition, and the uniformity of the distribution of the zinc powder particles over the entire treated surface.

During the galvanising processes used in production, the mass transfer of zinc to the steel surface is accomplished through a contact method - by baking the powder particles onto the zinc surface, and a non-contact method, through the gas phase - due to the high elasticity of zinc vapours at galvanising temperatures (Proskurkin, Gorbunov, 1972). It is also possible to condense zinc vapours on the treated surface due to the temperature difference between the powder medium and the workpiece. In this case, the reaction diffusion processes in the "steel - vapour phase" and "steel - solid or liquid zinc" boundaries occur differently. The prevalence of one galvanising mechanism or another is connected with the magnitude and direction of the temperature gradient in the reaction zone, the number and size of zinc powder particles, and the inert component in the saturating mixture.

Thus, problems concerning the ability to reproduce the galvanising results in mass production are linked with changes in a variety of parameters, associated both with the composition of the saturating mixtures and the complex coating-formation mechanism.

To increase the stability of the powder-saturating-mixture's dispersion composition and improve the consistency of galvanizing results, saturating mixtures have been obtained through the hydrothermal treatment of zinc powder (Galin et al., 1988). The high stability of this powder against sintering, the fusing of particles, and baking onto the zinc surface is provided due to the synthesis of zinc particles of a nanocrystalline structure consisting of dispersed zinc oxide which occurs on the surface.

It was shown in (Galin, Voroshnin, 1996), based on model experiments, that during the galvanising process in nanocrystallised zinc powders, the main mechanism for the formation of a diffusion zinc coating is a contact mass transfer 
of zinc from the surface of particles to the surface of the workpiece. The process is regulated by a small set of parameters - the temperature and duration of the contact of the workpiece with the saturating medium. The exclusion of unwieldy evaporation and condensation processes allows the production effectiveness of diffusion galvanising in powders to be significantly improved.

The method of contact galvanising in nanocrystallised zinc powders is characterised by the ability to utilise powder for galvanising in a wide temperature range - from $400^{\circ} \mathrm{C}$ to $600^{\circ} \mathrm{C}$. At temperatures above $550^{\circ} \mathrm{C}$, the galvanising time to produce $50-100 \mu \mathrm{m}$ coatings is a few minutes. The saturation ability of nanocrystallised powders is mainly determined by the amount of saturating mixture in the galvanising chamber, the galvanising temperature and time. In practice, the coating begins to form when the workpieces are heated to the optimum galvanising temperature. The speed of the process is limited only by the rate of heating the treated workpieces up to the galvanising temperature.

The homogeneity of the saturating mixtures makes it possible to create cyclic and continuous-action high-performance equipment (up to $1 \mathrm{t} / \mathrm{h}$ ) for the thermal diffusion galvanising of metalware, pipes, and bulk workpieces, with dimensions that exceed the typical sizes of hot-dip galvanising baths. The increased corrosion and mechanical properties of the coatings obtained in nanocrystallised zinc powders allow us to expand the usable range of diffusion zinc coatings in aggressive corrosive mediums for critical parts, such as threaded tubing connections of pumping and compression pipes (PCP) in the oil industry. The patented method for large-scale diffusion galvanising of PCP couplings using nanocrystallised zinc powders has been successfully implemented at the plants of PNTZ JSC (Russia), SinTZ PJSC (Russia), and KSP Steel LLP (Kazakhstan).

Diffusion galvanising in nanocrystallised zinc powders can be performed in sealed containers with or without rotation. However, the lack of a clear understanding of the mechanism for the formation of diffusion zinc coatings based on the use of nanocrystallised zinc powder complicates the extensive mastering of the new method and, most importantly, the calculation of the process parameters of the newly-created equipment.

This article presents comparative studies of the saturating capacity of nanocrystallised zinc powders in the initial state and after repeated use in a production environment. The same powders were used to estimate the mass transfer from the powder particles to the steel surface based on a layer-to-layer powder microanalysis.

\section{Materials and methods}

Disk-shaped samples of steel 20 (chemical composition according to GOST 1050:2013) with a diameter of $36 \mathrm{~mm}$ and a thickness of $3 \mathrm{~mm}$ were chosen for the study. Nanocrystallised zinc powder produced according to the procedure in (Galin, Voroshnin, 1996) was used as the saturating medium for diffusion
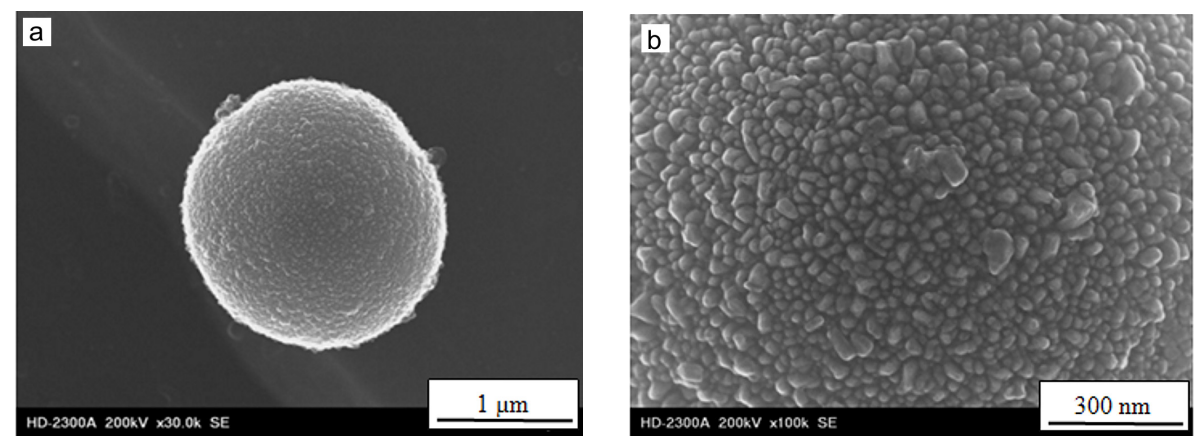

galvanising. Figure 1 shows an electron microscopic image of a nanocrystallised powder particle with nanocrystalline zinc oxide on the surface. The powder was studied both in its initial state and after repeated use (Table 1). The compositions of the powders used were determined through atomic emission spectrometry on an iCAP 6500 Duo spectrometer (Table 1). The bulk density of the studied powders was determined according to details provided in the

Fig. 1. Microstructure of zinc powder particle: a) STEM image, b) the nanocrystallised particle's shell (Galin, Zakharyevich, Ruschitz, 2016) 


\section{口:: technical Eםtransactions}

Table 1. Composition and density of the nanocrystallised powders used for galvanising

\begin{tabular}{|c|c|c|c|c|}
\hline \multirow{2}{*}{ Powder mixture } & \multicolumn{3}{|c|}{ Composition, wt. \% } & \multirow{2}{*}{ Bulk density, $\mathbf{g} / \mathbf{c m}^{3}$} \\
\cline { 2 - 4 } & Zn & Zno & Fe & \\
\hline Initial powder & 92.0 & 8.0 & 0.0 & 3.7 \\
\hline After repeated use & 65.0 & 34.0 & 1.0 & 2.3 \\
\hline
\end{tabular}

Galvanising by rotation was performed in sealed containers in a thermal diffusion galvanising plant in accordance with details provided in standard (GOST $\mathrm{R}$ 9.316-2006). The processing time varied from 0.5 to 3 hours; the galvanising temperatures were $400^{\circ} \mathrm{C}, 430^{\circ} \mathrm{C}$, and $450^{\circ} \mathrm{C}$.

To apply coatings in static conditions, we used a sealed container in which the samples for galvanizing and the saturating mixture were placed, so that the samples and the mixture completely occupied the space inside the container. The air was then pumped out to a residual pressure of $10^{-3} \mathrm{~atm}$. The container was filled with argon with an excess pressure of $0.05-0.10 \mathrm{~atm}$ and placed in a shaft furnace. The final temperature of the furnace was regulated through measurement of the temperature near the sample placed in the centre of the container.

The thickness of the obtained coatings was evaluated metallographically using a Zeiss Axio Observer D1m optical microscope. The mass transfer intensity was estimated by measuring the change in the weight of the samples during the galvanising process relative to the total surface area of the galvanised samples. X-ray phase analysis was performed with a Rigaku Ultima IV diffractometer. The surfaces and sections of the samples were studied on a JEOL JSM-6460 LV scanning electron microscope (SEM) equipped with an Oxford Instruments energy dispersive spectrometer (EDS) for chemical microanalysis.

We conducted model experiments to study the composition of the powder adjacent to the galvanised surface and clarify the nature of the contact interaction of the powder particles of the saturating mixture and the steel surface. The studied powders (initial and after repeated use see Table 1) were poured into the cavity of the cylinders made of steel 20 (Fig. 2). The cylinders were placed in the centre of the sealed galvanising container under static conditions and the container was filled with powder of the same composition. The dimensions of the cylindrical

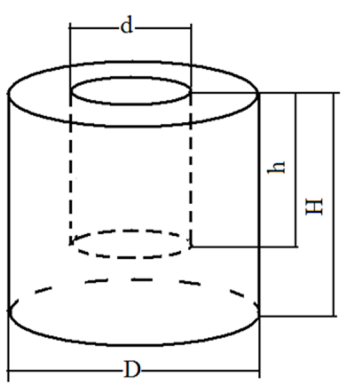
cavity and the temperature conditions of galvanising were chosen experimentally so as to exclude mixing of the surface powder layers during the study. The galvanising temperature was $550^{\circ} \mathrm{C}$, with a time of $1 \mathrm{~h}$. We then determined the chemical composition of the powders placed in the cylindrical cavities using layer-to-layer microanalysis on an SEM with an EDS attachment.

The following experiments were performed to determine the influence of the zinc vapour phase on the mass transfer processes in nanocrystallised zinc powders (see Table 2). Fifty grams of pure InstZn-325 zinc powder with a particle size of over $100 \mu \mathrm{m}$ was thoroughly mixed with 300 grams of nanocrystallised powder with a particle size of less than $40 \mu \mathrm{m}$ that had previously been used several times. Heat treatment of the compound powder was then performed in the sealed container. The temperature conditions were chosen taking into account the results of previous experiments (Galin, Voroshnin, 1996), which showed high
Fig. 2. Scheme and dimensions of two cylindrical steel samples made for the model experiments. The samples differ by their inner diameter 
stability of the dispersion composition of nanocrystallised zinc powders against sintering and melting of particles up to $650^{\circ} \mathrm{C}$. The particle size after heating was determined using the sieve analysis method (GOST 18318-94).

Table 2. The change in the dispersion and amount of the powder particles after galvanising at $550^{\circ} \mathrm{C}$ for 2.5 or $3.5 \mathrm{~h}$.

\begin{tabular}{|l|c|c|c|c|c|}
\hline \multirow{2}{*}{$\begin{array}{c}\text { Powder composition } \\
\text { and galvanising } \\
\text { mode }\end{array}$} & \multicolumn{3}{|c|}{ Granulometric composition of the powder } & \multirow{2}{*}{ Total amount } \\
\cline { 2 - 5 } & Max. $40 \mu \mathrm{m}$ & $40-63 \mu \mathrm{m}$ & $63-100 \mu \mathrm{m}$ & Min. $100 \mu \mathrm{m}$ & \\
\hline $\begin{array}{l}300 \text { g of used powder } \\
\text { with particle size } \\
<40 \mu \mathrm{m} ; 550^{\circ} \mathrm{C} / 3.5 \mathrm{~h}\end{array}$ & $300 \mathrm{~g}$ & $0 \mathrm{~g}$ & $0 \mathrm{~g}$ & $0 \mathrm{~g}$ & $300 \mathrm{~g}$ \\
\hline $\begin{array}{l}300 \mathrm{~g} \text { of used powder } \\
\text { with particle size } \\
<40 \mu \mathrm{m} \text { and } 50 \mathrm{~g} \\
\text { of pure zinc particles } \\
>100 \mu \mathrm{m} ;\end{array}$ & $128 \mathrm{~g}$ & $188 \mathrm{~g}$ & $2 \mathrm{~g}$ & $32 \mathrm{~g}$ & $350 \mathrm{~g}$ \\
$550^{\circ} \mathrm{C} / 2.5 \mathrm{~h}$ & $202 \mathrm{~g}$ & $145 \mathrm{~g}$ & $3 \mathrm{~g}$ & $0 \mathrm{~g}$ & $350 \mathrm{~g}$ \\
\hline $\begin{array}{l}300 \mathrm{~g} \text { of used powder } \\
<40 \mu \mathrm{m} \text { and } 50 \mathrm{~g} \\
\text { of pure zinc }>100 \mu \mathrm{m} ; \\
550^{\circ} \mathrm{C} / 3.5 \mathrm{~h}\end{array}$ & & & & & \\
\hline
\end{tabular}

\section{Results and discussion}

\subsection{Galvanising by rotation in powders of different composition}

The change in the sample weight before and after galvanising in the initial nanocrystallised powder at the temperature of $400^{\circ} \mathrm{C}$ in a rotary furnace for $1 \mathrm{~h}$ and $1 \mathrm{~h} 15$ min turned out to be negative and amounted to $-0.57 \mathrm{~g} \cdot \mathrm{m}^{-2}$ and $-0.70 \mathrm{~g} \cdot \mathrm{m}^{-2}$, respectively. With an increase in the galvanising time to $1 \mathrm{~h} 30 \mathrm{~min}$ and above, the change in the sample weight was positive: $+0.76 \mathrm{~g} \cdot \mathrm{m}^{-2}$. The chemical composition on the surface of the test samples was analysed in three areas (Table 3). It has been established that the composition of the formed coating corresponds to a solid solution of zinc in the $\alpha$-phase of iron. Similar results were obtained at low galvanising temperatures of $320-350^{\circ} \mathrm{C}$ for $2.5 \mathrm{~h}$ in a rotary furnace (Galin, Voroshnin, 1996).

Table 3. EDS results of three micro-areas of the galvanised specimen surface

\begin{tabular}{|c|c|c|c|}
\hline \multirow{2}{*}{ Spectrum } & \multicolumn{2}{|c|}{ Composition, at. \% } & \multirow{2}{*}{ Total } \\
\cline { 2 - 3 } & Fe & Zn & 100.0 \\
\hline 1 & 99.5 & 0.5 & 100.0 \\
\hline 2 & 99.5 & 0.5 & 100.0 \\
\hline 3 & 99.8 & 0.2 & 100.0 \\
\hline Average & 99.6 & 0.4 & \\
\hline
\end{tabular}

A further increase in the galvanising time and temperature leads to the formation of intermetallic phases in the coating according to the Fe- $\mathrm{Zn}$ state diagram ( $\Gamma$-phase of $\mathrm{Fe}_{11} \mathrm{Zn}_{40}$ and $\delta$-phase of $\mathrm{FeZn}_{7-10}$ ) (Fig. 3). With an increase in the duration of galvanising ( $2 \mathrm{~h} 20 \mathrm{~min}, 2 \mathrm{~h} 30 \mathrm{~min}, 3 \mathrm{~h} 20 \mathrm{~min}$ and $4 \mathrm{~h}$ ) at the same temperature of $430^{\circ} \mathrm{C}$ with a simultaneous increase in the coating thickness, we observed an increase in the concentration of zinc in the $\delta$-phase (Fig. 4).

According to the obtained data, the zinc coating layer contains the $\Gamma$-phase $\left(\mathrm{Fe}_{3} \mathrm{Zn}_{10}\right.$-type compound) in the form of a thin interlayer with an average thickness of $2 \mu \mathrm{m}$ at the surface of the steel base (Fig. 3). In this case, the thickness of the $\Gamma$-phase layer has a weak dependence upon the galvanising mode. Next there is an intermediate layer, which cannot be detected during etching $\left(\delta_{1}\right.$-phase), with 
a zinc content of 79 to 88.5 wt. \%. According to the Fe-Zn state diagram, this corresponds to the area of the mixture of $\Gamma$ - and $\delta$-phases. The thickness of the intermediate layer ranges from 1.5 to $10 \mu \mathrm{m}$ and significantly depends on the galvanising mode. On the outer surface, the zinc coating contains a $\delta$-phase layer ( $\mathrm{FeZn}_{7}$-type compound) with a characteristic columnar structure. The content of zinc in the $\delta$-phase is from 88.5 to $93 \mathrm{wt}$. \%. The arrangement of the intermetallic phase layers in the zinc coating is shown in Fig. 3. It should be noted that, according to the data available in the literature, no similar coating structure is observed when traditional galvanising methods are used.

For the process of galvanising in the same modes as those described above, but using the powder after repeated use, there are generally similar results; however, with a two-hour delay at the beginning of the intermetallic phase formation (Table 4).

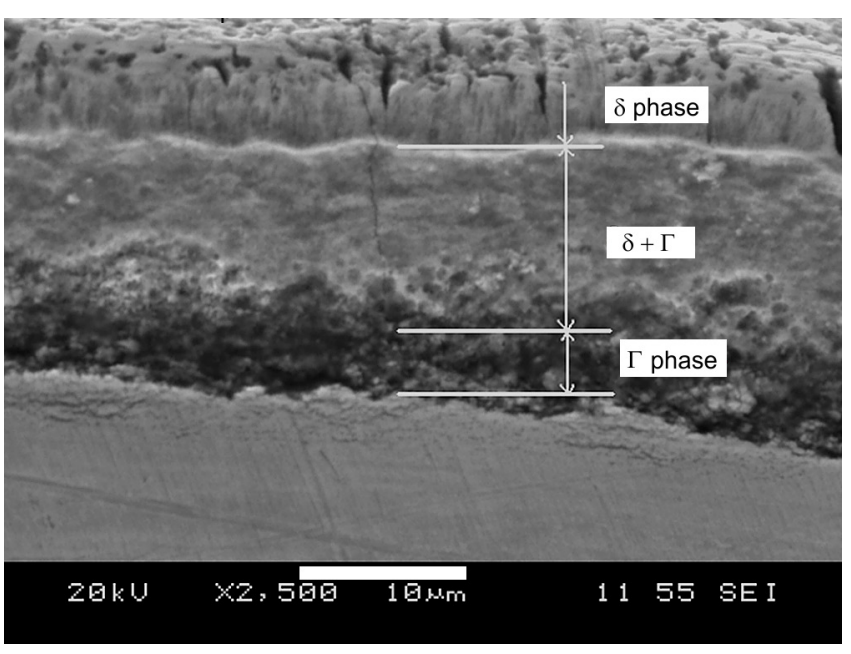

Fig. 3. Cross-section microstructure of the galvanised coating, SEM image powder due to a decrease in its bulk density possibly contributes to a decrease in the saturating capacity of the powder during diffusion galvanising. The thickness and phase composition of zinc coatings obtained in a rotary furnace depending on the galvanising modes for various powders are shown in Table 4.
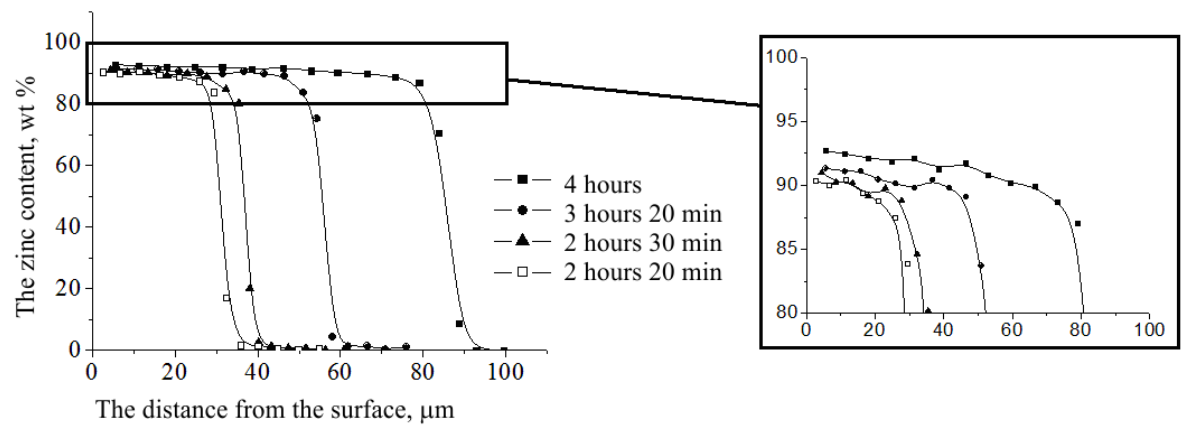

Table 4. Thickness of the phase layers in the coatings for the galvanising temperature of $430^{\circ} \mathrm{C}$. * Time of heating to the galvanising temperature: 1 hour; (-) no coating detected

\begin{tabular}{|c|c|c|c|c|c|}
\hline \multirow{2}{*}{\multicolumn{2}{|c|}{ Galvanising mode* }} & \multicolumn{4}{|c|}{ Galvanising powder mixture } \\
\hline & & \multicolumn{2}{|c|}{ Initial powder } & \multicolumn{2}{|c|}{ After repeated use } \\
\hline Temperature, ${ }^{\circ} \mathrm{C}$ & Time, $\mathrm{h}$ & Phase & Thickness, $\mu \mathrm{m}$ & Phase & Thickness, $\mu \mathrm{m}$ \\
\hline 430 & 0.5 & $\delta$ & 15 & \multicolumn{2}{|c|}{$(-)$} \\
\hline 430 & 1 & $\delta$ & 20 & \multicolumn{2}{|c|}{$(-)$} \\
\hline 430 & 1.2 & $\delta$ & 25 & \multicolumn{2}{|c|}{$(-)$} \\
\hline 430 & 1.3 & $\delta$ & 32 & \multicolumn{2}{|c|}{$(-)$} \\
\hline 430 & 1.5 & $\xi\left(F e Z n_{13}\right)+\delta$ & 37 & \multicolumn{2}{|c|}{$(-)$} \\
\hline 430 & 1.7 & $\xi\left(F e Z n_{13}\right)+\delta$ & 45 & \multicolumn{2}{|c|}{$(-)$} \\
\hline 430 & 1.8 & $\xi\left(F e Z n_{13}\right)+\delta$ & 50 & \multicolumn{2}{|c|}{$(-)$} \\
\hline 430 & 2 & $\xi\left(F e n_{13}\right)+\delta$ & 80 & \multicolumn{2}{|c|}{$(-)$} \\
\hline 430 & 2.5 & $\xi\left(F e Z n_{13}\right)+\delta$ & 92 & $\begin{array}{c}\delta+\Gamma \\
\delta\end{array}$ & $\begin{array}{l}25 \\
33\end{array}$ \\
\hline 430 & 2.8 & $\xi\left(F e n_{13}\right)+\delta$ & 97 & $\delta$ & 35 \\
\hline 430 & 3 & $\xi\left(\mathrm{FeZn} \mathrm{n}_{13}\right)$ & 102 & $\delta$ & 50 \\
\hline
\end{tabular}

It should be noted that after the thermal diffusion galvanising of the steel samples in nanocrystallised zinc powders, an easily-removable powder layer with a thickness of up to $0.5 \mathrm{~mm}$ remains on the sample surface, the amount of which
Fig. 4. Coating thickness and zinc distribution in the samples galvanised in various modes 
Fig. 5. XRD spectra of the phase composition of the galvanised steel surface: a) $\delta$-phase for coating thickness of $32 \mu \mathrm{m}, \mathrm{b}$ ) mixture of $\xi\left(\mathrm{FeZn}_{13}\right)$ and $\delta$-phase for coating thickness of $80 \mu \mathrm{m}$ a)

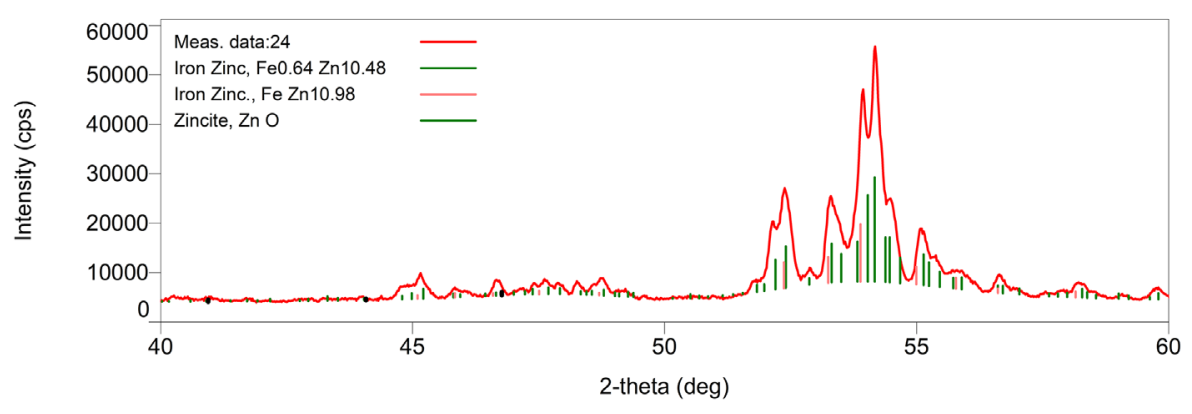

b)

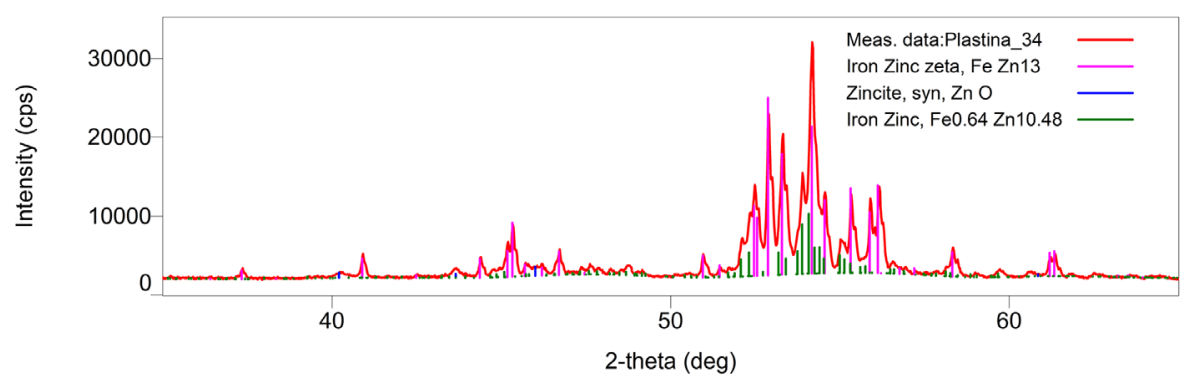

increases with an increase in the coating thickness. Chemical analysis of the residual powder composition performed in accordance with the procedure outlined in standard (GOST 12601-76) shows a decrease in the content of metallic zinc in the surface layer of the powder to 50-75 wt. \%.

Two typical XRD spectra of the phase composition of the galvanised steel surface are shown in Fig. 5. The results confirm the formation of the $\delta$-phase for a coating thickness of $32 \mu \mathrm{m}$ (see Table 4). For a coating thickness of $80 \mu \mathrm{m}$, a mixture of $\xi(F e Z n 13)$ and $\delta$-phase was detected.

According to the literature (Kubashevski, Alcock, 1979), the zinc diffusion coefficient in the vapour phase at its melting temperature is $3.7 \cdot 10^{-5} \mathrm{~m}^{2} \cdot \mathrm{s}^{-1}$. It follows that in the case of a mass transfer through the vapour phase, possible heterogeneities of the powder composition in terms of zinc with dimensions of around $0.5 \mathrm{~mm}$ should be homogenised during the galvanising process within a few seconds. Since, according to the experiment results, this does not happen, it can be assumed that the mass transfer of zinc in the powder occurs from particle to particle. The results of subsequent experiments and calculations confirm this hypothesis.

\subsection{Model experiments covering the layer-to-layer microanalysis of powders}

Figures 6 and 7 show the results of the layer-to-layer microanalysis of the initial nanocrystallised zinc powder placed in a cylindrical cavity of Type 1 samples (see Fig. 2), and nanocrystallised zinc powder after repeated use placed in a cylindrical cavity of Type 2 samples (see Fig. 2), respectively.

The data of the linear (layer by layer) microanalysis of the saturating mixtures show that during thermal diffusion galvanizing of a steel sample, in addition to the enrichment of steel with zinc, there is a decrease in the concentration of zinc in the powder layers in contact with the metal surface and the mass transfer of iron to the saturating mixture. At the same time, the thickness of the powder-enriched and zinc-poor powder layer is about $1.0 \mathrm{~mm}$ for the initial saturating mixture, and about $3 \mathrm{~mm}$ for the mixture after repeated use (Table 5 and Table 6). 
Table 5. Results of the linear (layer by layer) microanalysis of the initial zinc powder

\begin{tabular}{|c|c|c|c|}
\hline \multirow{2}{*}{ Spectrum } & \multicolumn{3}{|c|}{ Composition of specimen (1), at. \% } \\
\cline { 2 - 4 } & $\mathbf{0}$ & Fe & Zn \\
\hline 1 & 21.0 & 0.2 & 78.8 \\
\hline 2 & 14.2 & 0.1 & 85.7 \\
\hline 3 & 18.9 & 0.1 & 81.0 \\
\hline 4 & 12.3 & 0.1 & 87.6 \\
\hline 5 & 12.5 & 0.0 & 87.4 \\
\hline 6 & 7.0 & 0.1 & 92.9 \\
\hline 7 & 4.1 & 0.0 & 95.8 \\
\hline 8 & 14.1 & 0.2 & 85.6 \\
\hline 9 & 9.5 & 0.2 & 90.2 \\
\hline 10 & 14.1 & 0.1 & 85.8 \\
\hline
\end{tabular}

Table 6. Results of the linear (layer by layer) microanalysis of the zinc powder after repeated use

\begin{tabular}{|c|c|c|c|}
\hline \multirow{2}{*}{ Spectrum } & \multicolumn{3}{|c|}{ Composition of specimen (2), at. \% } \\
\cline { 2 - 4 } & 0 & Fe & Zn \\
\hline 1 & 21.9 & 3.1 & 75.0 \\
\hline 2 & 27.1 & 2.8 & 70.1 \\
\hline 3 & 23.2 & 2.2 & 74.6 \\
\hline 4 & 24.7 & 2.0 & 73.2 \\
\hline 5 & 21.4 & 1.1 & 77.5 \\
\hline 6 & 26.5 & 1.3 & 72.2 \\
\hline 7 & 16.6 & 1.8 & 81.6 \\
\hline 8 & 20.4 & 1.6 & 78.0 \\
\hline 9 & 21.4 & 1.5 & 77.2 \\
\hline 10 & 20.7 & 1.1 & 78.2 \\
\hline 11 & 18.7 & 1.1 & 80.2 \\
\hline
\end{tabular}

It can be assumed that the mass transfer of zinc and counter mass transfer of iron occur due to the diffusion of zinc and iron atoms through the surface film of zinc oxide on the powder particles. On the basis that the total thickness of the iron-enriched and zinc-depleted powder layer may depend on the time $x \sim \sqrt{2 D \tau}$, where $D$ is the effective diffusion coefficient and $\tau$ is the annealing time, we estimated the diffusion coefficient: $D \approx 1.4 \cdot 10^{-10} \mathrm{~m}^{2} / \mathrm{s}$ for the original saturating mixture and $D \approx 1.2 \cdot 10^{-9} \mathrm{~m}^{2} / \mathrm{s}$ for the mixture after repeated use. In our previous work (Galin, Voroshnin, 1996), we experimentally obtained an equation to determine the diffusion coefficient of zinc in the surface layer of zinc oxide on zinc powder particles during the synthesis of the oxide layer: $D_{\mathrm{zn}}=2.08 \cdot 10^{-4} \times \exp \left(-11.0 \cdot 10^{3} / \mathrm{T}\right) \mathrm{m}^{2} / \mathrm{s}$. The diffusion coefficient of zinc in zinc oxide calculated by this formula for the model experiment temperature of $550^{\circ} \mathrm{C}$ is $1.9 \cdot 10^{-10} \mathrm{~m}^{2} / \mathrm{s}$, i.e. the nanocrystalline structure of the surface film on the zinc powder particles is not subjected to significant changes during the galvanising process. The obtained values of the diffusion coefficients are several orders of magnitude higher than the literature values (Izvekov, Gorbunov, 1969), which is explained by the defectiveness of the oxide film on the zinc powder particles.

It was shown in previous research (Galin, Shaburova, Zakharyevich, 2016) that during the galvanising process in nanocrystallised zinc powders alloyed with iron, the thickness of the $\alpha$-solid solution of zinc in iron increases with an increase in the iron content in the powder. For example, for a sample galvanised in the powder of 2 wt.\% Fe, 90 wt.\% Zn, and 8 wt.\% ZnO, the thickness of

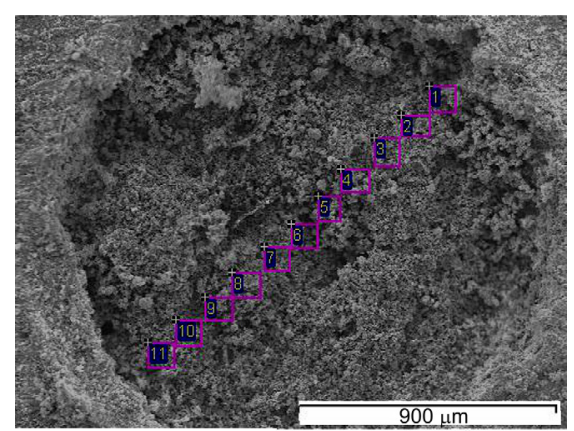

Fig. 6. Linear (layer by layer) microanalysis of the initial zinc powder (specimen Type 1 in Fig. 2)

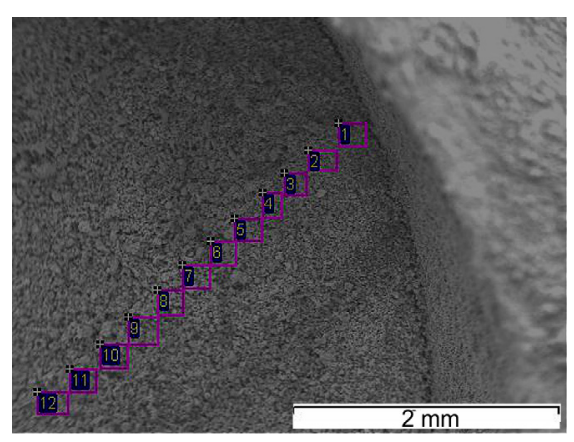

Fig. 7. Linear (layer by layer) microanalysis of the zinc powder of repeated use (specimen Type 2 in Fig. 2) 
this layer reaches $80 \mu \mathrm{m}$, which exceeds the thickness of the formed layer of intermetallic iron-zinc phases.

Considering that, after repeated use, the iron content in the surface areas of the powder is an order of magnitude higher than in the initial powder, it can be assumed that the larger thickness of the solid solution during the galvanising process in the powders alloyed with iron is explained by a weaker diffusion flow of zinc in the boundary region due to a decrease in the zinc concentration drop at the "powder - steel" boundary and its higher diffusion coefficient in comparison with pure nanocrystallised powder.

\subsection{The influence of the vapour phase on the mass transfer processes during galvanising}

The mixtures of the studied powders consisted of $300 \mathrm{~g}$ of nanocrystallised powder and $50 \mathrm{~g}$ of pure zinc powder. The maximum evaporation surface for 50 grams of the pure powder, assuming that all the particles had a diameter of $100 \mu \mathrm{m}$, was $4.2 \cdot 10^{3} \mathrm{~cm}^{2}$. According to data in the literature (Kazenas, Astakhova, Penkina, 1998), the evaporation rate of zinc at the melting temperature is $27.8 \cdot 10^{-4} \mathrm{~g} \cdot \mathrm{cm}^{-2} \mathrm{~s}^{-1}$ and is therefore $12 \cdot 10^{-3} \mathrm{~g} \cdot \mathrm{cm}^{-2} \mathrm{~s}^{-1}$ at $852 \mathrm{~K}\left(579^{\circ} \mathrm{C}\right)$. Thus, pure zinc powder without surface contamination should evaporate in the first seconds of heating once the melting point of zinc is exceeded. These theoretical calculations are confirmed by the experimental results given in Table 2. As can be seen from Table 2, as a result of holding the composite powder at $550^{\circ} \mathrm{C}$ for 3.5 hours, the particles of the pure zinc powder "evaporated" into the volume of the nanocrystallised zinc powder.

It should be assumed that the absence of particles over $100 \mu \mathrm{m}$ in the composite powder at the temperature above the melting point of zinc is connected with the fact that the pure zinc particles did not adhere to the particles with a nanocrystalline shell of zinc oxide. At the same time, there was neither liquid zinc wetting of the nanocrystalline protrusions of the $\mathrm{ZnO}$ layer on the surface of the particles, nor condensation of zinc vapours on these protrusions, since the vapour condensation on convex surfaces requires a larger supersaturation of the zinc vapour.

To clarify the possible condensation of zinc vapours on the convex and concave surfaces of zinc oxide, we evaluated the influence of the radius of the surface curvature of pure zinc on the equilibrium vapour pressure of $\mathrm{Zn}$ in a temperature range corresponding to the galvanising process $(T=573-923 \mathrm{~K})$.

The equilibrium vapour pressure of zinc $P_{0}$ over the flat surface of pure zinc is calculated by the following formulas (Kubaschevski, Alcock, 1979):

at temperature $T$ below the melting point of zinc $T_{m}(\mathrm{Zn})=692.5 \mathrm{~K}$

$$
\lg P_{o}=-6850 / T+11.24-0.755 \cdot \lg T[\mathrm{~mm} \mathrm{Hg}],
$$

and at $T>T_{m}$

$$
\lg P_{o}=-6220 / T+12.34-1.255 \cdot \lg T[\mathrm{~mm} \mathrm{Hg}],
$$

where $1 \mathrm{~mm} \mathrm{Hg}=133.322 \mathrm{~Pa}$.

The vapour pressure over the convex or concave surface is determined by the Gibbs-Thomson formula, also called the Kelvin formula (Adamson, Gast, 1997):

$$
P_{\mathrm{Zn}}(r, T)=P_{o} \exp \left(-\frac{2 \sigma_{\mathrm{Zn}} \varpi_{\mathrm{Zn}}}{R T r}\right),
$$

where $\sigma_{\mathrm{zn}}\left[\mathrm{Jm}^{-2}\right]$ is the surface energy of zinc, $\varpi_{\mathrm{zn}}\left[\mathrm{cm}^{3} \mathrm{~mol}^{-1}\right]$ is its molar volume and $R\left[\mathrm{JK}^{-1} \mathrm{~mol}^{-1}\right]$ is the universal gas constant. Here, it is assumed for simplicity that the radius $r$ is equal to the radius of the curved surface. The "+" sign in formula (3) 
refers to the case of a convex surface (positive curvature, $r>0$ ), i.e. for a nanocrystal surface, and the "-" sign corresponds to a concave surface (negative curvature, $r<0$ ), i.e. for the gap between the nanocrystals (see Fig. 1, b).

The molar volume of zinc is determined as:

$$
\omega_{\mathrm{Zn}}=\mu_{\mathrm{Zn}} / \rho_{\mathrm{Zn}}
$$

where $\mu_{\mathrm{zn}}=65.4 \mathrm{~g} / \mathrm{mol}$ is the molecular weight; $\rho_{\mathrm{zn}}$ is the zinc density, $\mathrm{g} / \mathrm{cm}^{3}$.

For solid zinc $\left(T<T_{m}\right)$, the density is constant $\rho_{\mathrm{zn}}=7.14 \mathrm{~g} / \mathrm{cm}^{3}$, while for molten zinc (at $T>T_{m}$ ), it is dependent upon the temperature (Brandes, Brook, 1992):

$$
\rho_{\mathrm{Zn}}=6.575-1.1 \cdot 10^{-3}\left(T-T_{m}\right)\left[\mathrm{g} / \mathrm{cm}^{3}\right] \text {. }
$$

For solid zinc, the surface energy is constant, $\sigma_{\mathrm{zn}}=0.828 \mathrm{~J} / \mathrm{m}^{2}$ [25], while for molten zinc, it is dependent upon the temperature (Missol, 1975):

$$
\sigma_{\mathrm{Zn}}=0.782-0.17 \cdot 10^{-3}\left(T-T_{m}\right)\left[\mathrm{J} / \mathrm{m}^{2}\right] .
$$

The calculation results for the concave surface of solid zinc ( $T<T_{m}, \sigma_{\mathrm{zn}}=$ const, $\rho_{\mathrm{zn}}=$ const) are shown in Fig. 8.

It is clear from Fig. 8 that the increase in the equilibrium pressure for solid zinc becomes noticeable when the radius of the protrusions is less than $50 \mathrm{~nm}$ and most significant when approaching the melting point of zinc. The increase in $P_{\mathrm{zn}}(r)$ itself in comparison to the equilibrium pressure above the flat surface $P_{0}$ is small: for example, at $T=T_{m}$ and $r=5 \mathrm{~nm}$, it is $\approx 14 \mathrm{~Pa}$. However, in relative terms, this increase in the vapour pressure is significant (by $35 \%$ from $P_{0}=22 \mathrm{~Pa}$ for the flat surface to $P_{\mathrm{zn}}=36 \mathrm{~Pa}$ for the convex surface of a specified radius).

The results of the calculations by formulas (1)-(6) for the temperature range of 573-923 K (below and above the melting point of zinc) for the convex zinc surface are shown in Fig. 9.

It follows from Fig. 9 that the increase in the equilibrium pressure for zinc is noticeable when the radius of the protrusions is less than $50 \mathrm{~nm}$ and most significant at high temperatures. Thus, at $T=923$ $\mathrm{K}$ for the protrusion radius of $r=5 \mathrm{~nm}$, the zinc vapour pressure increases from $P_{0}=3800 \mathrm{~Pa}$ for the flat surface to $P_{\mathrm{zn}}=5560 \mathrm{~Pa}$, i.e. by $46 \%$.

For concave surfaces, the calculation was also made in the temperature range of 573-923 $\mathrm{K}$ (below and above the melting point of zinc). The results are shown in Fig. 10.

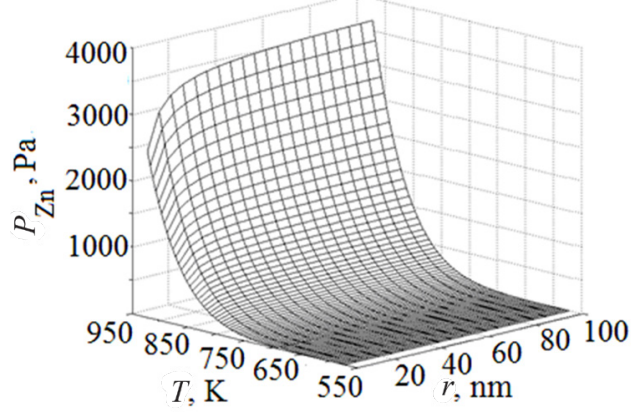

Fig. 8. The dependence of the equilibrium vapour pressure of zinc above the surface of the solid metal on the temperature and radius of the concave surface $(r<0)$
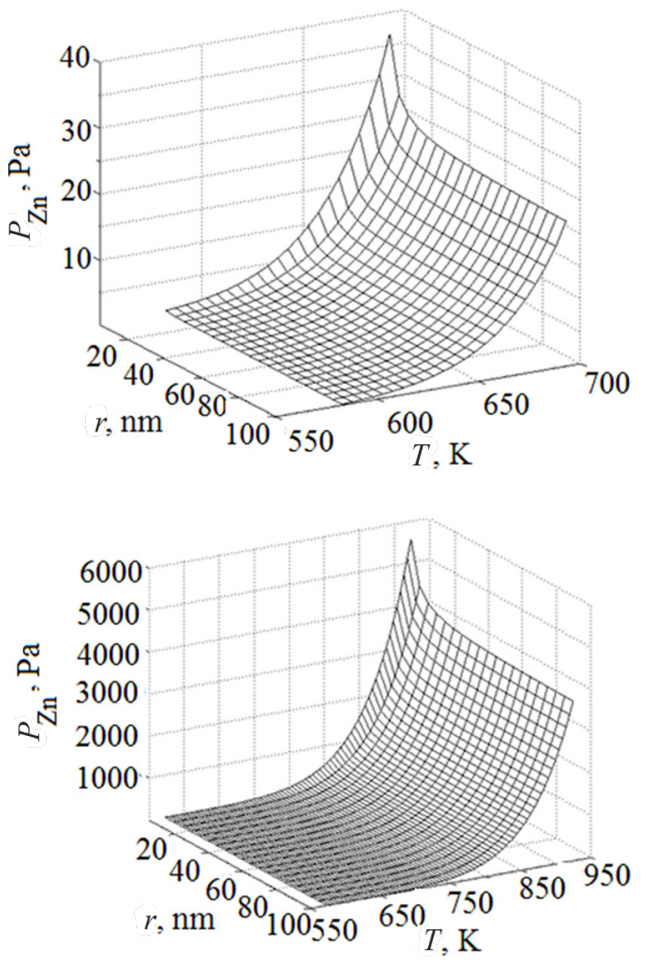

Fig. 9. The dependence of the equilibrium vapour pressure of zinc above the surface of solid (at $T<T_{m}$ ) and liquid (at $T>T_{m}$ ) zinc on the temperature and radius of the convex surface $(r>0)$ 
As can be seen from Fig. 10, at $T>T_{m}$, there is a significant decrease in the equilibrium pressure when the radius of the concave sections is less than $50 \mathrm{~nm}$, and this effect is most significant at high temperatures. Thus, at $T=923 \mathrm{~K}$ for the radius of the concave sections $r=5 \mathrm{~nm}$, the zinc vapour pressure decreases from $P_{0}=3800 \mathrm{~Pa}$ for the flat surface to $P_{\mathrm{zn}}=2500 \mathrm{~Pa}$, i.e. by $34 \%$.

The equilibrium pressure of saturated zinc vapours for the flat surface $P_{0}$ at the model experiment temperature of $550^{\circ} \mathrm{C}$ is $533 \mathrm{~Pa}$. Equilibrium pressures for the protrusions and concave sections with radii of $5 \mathrm{~nm}$ are $746 \mathrm{~Pa}$ and $375 \mathrm{~Pa}$, respectively. Thus, the difference in the equilibrium pressures relative to the flat surface is $158 \mathrm{~Pa}$, and $371 \mathrm{~Pa}$ relative to convex surfaces.

It is natural to assume that the pure-powder particles evaporate and the zinc vapours condense in the concave sections of the nanocrystallised powder particles, increasing the number of particles over $40 \mu \mathrm{m}$.

It should be noted that the required pressure drop of metal vapours in the area of the evaporation and condensation of metals for the thermal vacuum deposition method is 100-200 Pa (Hass, Thuns, 1969).

The features of the formation of diffusion layers during zinc galvanising in nanocrystallised zinc powders were studied in previous research (Galin, Astrejko, 2008). It was shown that the growth of the diffusion coating proceeds both inwards and outwards due to the diffusion of zinc and iron atoms in opposite directions: the thickness of the coating significantly exceeds the change in the dimensions of the samples during processing. For specimens made from steel St. 3 galvanised at $600^{\circ} \mathrm{C}$ for 1 hour, the coating thickness was $140.1 \mu \mathrm{m}$, and an increase in the size of the sample was $49.0 \mu \mathrm{m}$. Specimens of steel St.3 galvanised at $450^{\circ} \mathrm{C}$ for 1 hour had a coating thickness of $45.0 \mu \mathrm{m}$ and showed an increase in the size of the sample of $8.0 \mu \mathrm{m}$. Model experiments also showed the presence of contact mass transfer of iron to the saturating medium during the galvanising process.

\section{Conclusions}

Zinc coating layers are formed sequentially during the galvanising process in nanocrystallised powders according to the iron-zinc equilibrium diagram: $\alpha$-phase $\rightarrow \Gamma$-phase $\rightarrow \delta_{1}$-phase $\rightarrow \delta$-phase $\rightarrow \zeta$-phase $\left(F e Z n_{13}\right)$. During the process, there is a counter mass transfer of iron from the saturable surface, and zinc from the saturating medium to the workpiece surface. The model experiments show that the mass transfer of zinc occurs through the nanocrystalline shell of $\mathrm{ZnO}$ on the powder particles, then through the contact points of the particles between each other and the saturable steel surface. The calculations of the zinc vapour pressure indicate the possible mass transfer of zinc from the convex sections of zinc oxide nanocrystals to the concave surfaces. This prevents sintering of the powder particles at the temperatures above the melting point of the powder material.

\section{References}

Adamson, A.W., Gast, A.P. (1997). Physical Chemistry of Surfaces, 6th edition, New York: John Wiley \& Sons.

Brandes, E.A., \& Brook, G.B. (Eds.). (1992). Smithells Metals Reference Book, $7^{\text {th }}$ edition, Oxford: Butterworth-Heinemann.

Galin, R.G., Sheinkman, A.I., Voroshnin, L.G., Ostryakov, V.V., Lunegov, B.N. (1988), Patent USSR No. 1534091 (application on 1988/04/27).

Galin, R.G., Voroshnin, A.L. (1996). New technology to manufacture powder saturation mixtures for diffusion galvanizing, Met Sci Heat Treat, 1, 21-25. DOI: 10.1007/BF01153868 
Galin, R.G., Astrejko, L.A. (2008). Diffusion zinc coatings obtained in hydrothermally treated powder media, Uprochniajushtee technologii i pokrytia, 1(28), 28-32.

Galin, R.G., Shaburova, N.A., Zakharyevich, D.A. (2016). Thermal diffusion galvanizing in ferriferous zinc powder, Mat Sci Forum, 870, 129-134. DOI: 10.4028/www.scientific.net/MSF.870.129

Galin, R.G., Zakharyevich, D.A., Ruschitz, S.V. (2016). Formation and Structure of Diffusional Zinc Coatings Formed in Nanocrystallized Zinc Powders, Mat Sci Forum, 870, 404-408. https://doi.org/10.4028/www.scientific.net/ MSF.870.404

GOST 19440-94. Metallic powders. Determination of apparent density. Part 1. Funnel method. Part 2. Scott volumeter method.

GOST 18318-94. (1997). Metallic powders. Determination of particle size by dry sieving.

GOST 12601-76 (cl. 4.3.2). (1976). Zinc powder. Specifications.

GOST R 9.316-2006. (2006). Unified system of corrosion and ageing protection. Thermodiffusion zinc coatings. General requirements and control methods.

GOST 1050:2013. (2013). Metal products from no alloyed structural quality and special steels. General specifications.

Hass, G., \& Thuns R.E. (Eds). (1969). Physics of Thin Films: Advances in Research and Development, vol. 5, New York: Academic Press.

Izvekov, V.I., Gorbunov, N.S. (1969). Radiometry of diffusion in metal compounds, Moscow: Nauka.

Kazenas, E.K., Astakhova, G.K., Penkina, T.N. (1998). On the issue of estimating the evaporation rate of metals, Metals, 1, 33-40.

Kubaschewski, O., Alcock, C.B. (1979). Metallurgical Thermochemistry, $5^{\text {th }}$ edition, Oxford: Pergamon Press.

Kuklik, V., Kudlacek, J. (2016). Hot-Dip Galvanizing of Steel Structures, Elsevier Ltd.

Missol, W. (1975). Energia powierzchni rozdziatu faz w metalach, Katowice: Wydawnictwo Śląsk.

Proskurkin, E.V., Gorbunov, N.S. (1972). Diffusion zinc coatings, Moscow: Metallurgiya.

Proskurkin, E.V., Gorbunov N.S. (1975). Galvanizing, Sherardizing and Other Zinc Diffusion Coatings. English translation by Hayler, D.E., UK: Technicopy Ltd. and Zinc Development Association, Stonehouse.

Revie, R.W., Uhlig, H.H. (2008). Corrosion and Corrosion Control: An Introduction to Corrosion Science and Engineering, $4^{\text {th }}$ edition, John Wiley \& Sons. 